\title{
Testing Three Hypotheses of the Contribution of Geometry and Migration Dynamics to Intestine Crypt Evolution
}

\author{
Arturo Araujo ${ }^{1}$, Albert Rübben ${ }^{2}$, Peter J Bentley ${ }^{1,3}$ and David Basanta ${ }^{4}$ \\ ${ }^{1}$ Braintree Ltd, London, United Kingdom \\ ${ }^{2}$ Euregio Skin Cancer Center, Department of Dermatology, University Hospital RWTH, Aachen, Germany \\ ${ }^{3}$ Department of Computer Science, UCL, London, United Kingdom \\ ${ }^{4}$ Moffitt Cancer Center, Florida, USA \\ a.araujo@braintree.com
}

\begin{abstract}
Intestinal glands in the small intestine and colon, or intestine crypts, are an important example of tissue homeostasis regulated by the extracellular environment. The crypts are invaginated structures made of a layer of cells that help absorb nutrients from passing food. However, they are continuously worn away by this process and are being continually renovated by stem cells at the bottom of the crypt. These stem cells divide to replace worn cells and may even displace other stem cells so that at a given time the whole crypt becomes monoclonal- a descendant of one single stem cell. From a theoretical standpoint, the time it takes to reach monoclonality is crucial to the understanding of colorectal cancer (CRC) as it offers a key metric for the establishment of cancer initiating mutations; however, the biggest biological contributor to this feature is highly debated. Three key hypotheses have been put forwards, which we investigated with ALife methods. We have abstracted key biological features and modelled them in a bottom-up Agent-Based Model that allowed us to study the biological first principles that rule the fixation of mutations, offering key spatial and temporal understanding of this process. Our results show that the number of basal stem cells have a direct influence on the fixations of mutations and suggesting a lesser role for extracellular influences, while proposing the existence of a threshold to the contribution of cell side displacement
\end{abstract}

\section{Introduction}

ALife methods aim to study life systems, their processes and evolution; offering us a glimpse of the hidden emergent dynamics and helping us gain an understanding of the complex information produced by these systems. In recent years, ALife has proven itself as a capable lens through which the most informative level of abstraction can be discovered and explored. By doing so, it is the intention that the organizing principle that make the biological processes can be elucidated and understood to make a more general theory of life neutral from the physical embodiment (Lenaerts, 2011). One of the biggest advantages of ALife techniques for the discovery of biological principles is the ability to use multiscale levels of abstraction to produce white-box models; giving a direct insight into the mechanisms at a resolution that is not obtainable through wet lab experiments alone (Kalmykov, 2015). This has led to the computational investigation of key

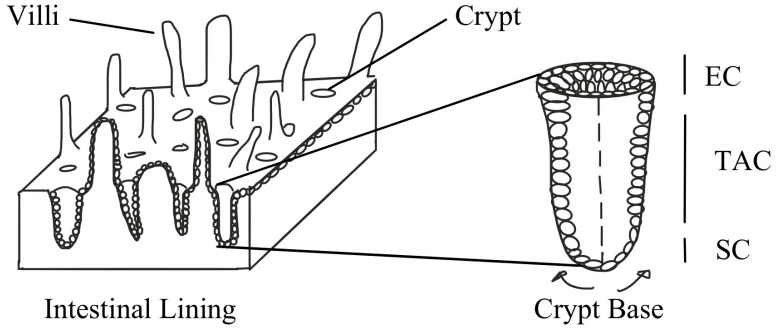

Figure 1: An intestinal crypt is an invagination in the inner lining of the intestine. Stem cells at the base constantly replenish the tissue.

biological behaviour such as tissue homeostasis (Sütterlin, 2009) and tissue architecture leading to organ formation (Galle, 2009). Because of this, ALife is ideal to address biological complex systems at the molecular, cellular and tissue level; and capable of shedding light on in vivo experiments that report seemingly different findings. One of such cases is the study of intestinal glands in the colon, or colon crypts: invaginations in intestinal tissue that help absorb nutrients as food passes through them (Figure 1). It has been shown that a niche of stem cells dividing from the bottom provides a continuous influx of new cells, and that eventually the dynamics (such as cell displacement), and the geometry of the crypt (such as the number of stem cells) will result in the whole crypt being the descendant of one such stem cells. However, there are different values reported for these contributors, and thus different theories on which one is the biggest contributor to the time to monoclonality: how long does it take for a percentage of crypts to become descendant of one single stem cell? This is important for the fixation of oncogenic mutations because it offers a metric by which these genetic alterations will spread through the crypt and make it a source of mutated cells in the intestine.

In this work, we use an agent-based model that simulates the known dynamics of homeostasis in the intestinal glands in the colon; but importantly has the sufficient plasticity to bridge the disparate in vivo and in vitro reported data into a baseline behaviour. With this baseline model, we investigate three hypotheses about the identity of the geometrical and dynamical constraints that has the largest effect on the fixation of mutations in the crypt: the total number of stem cells (Fletcher, 
2012), the proportion of side cell displacement (Ritsma, 2014) and the number of basal stem cells (Kozar, 2013).

\section{Background}

In the case of clinically relevant diseases, ALife has contributed much in helping extract the hidden evolutionary patterns and clinically relevant information in brain tumours (Swanson, 2003), and even shedding light on the origins of life (Kauffman, 2011, Froese, 2013). Great progress has been made in formalizing the simulation of complex biosystems and incorporating new nature-inspired computation back into the field (Andrews, 2010). As such, ALife methods have been used in the modelling of cancer (Araujo, 2010; Rübben \& Nordhoff, 2012) and had helped made advances in the way we discover novel therapeutic targets such as key switches in cell-cell communication (Bentley 2013). Because cancer is a process in which genetic mutations occurring at a subcellular level have repercussions at the cellular and tissue scale, modelling approaches that can handle multiple intracellular and extracellular factors acting on different time and space scales are needed (Rejniak, 2011). Specifically, agent-based modelling has been successful at modelling carcinogenesis; as was demonstrated by researchers simulating cells with internal genomes to create 3D structures, or artificial embryos, in which the process of cancer initiation could be abstracted and key mutations in the genomes studied (Fontana, 2010).

In the case of colon crypts, researchers have revisited through agent-based modelling the previously accepted theoretical model of colon crypt to understand the early progression of colorectal cancer (CRC), the second leading cause of cancer-related death in Europe and North America. In a recent study, after failing to recapitulate their data with it, researchers used their model to propose the need of cross-talk between cell types as a feature of colon crypt cycle models to account for the effects of variation of cell cycle rate, effectively opening new avenues of research in this area (Smallbone, 2014). Bravo and Axelrod measured the variation in stem cells, proliferating cells, and differentiated cells in multiple crypts in normal human biopsy specimens. With this data, they created an agent-based model that simulates the initiation and treatment of colon cancer. They focused on adenomas resulting from mutations either at the top or bottom of the crypt and provided a metric of the robustness with which crypts recover from chemotherapy and radiation scheduling protocols (Bravo, 2013).

Regarding the crypt geometry, initial advances were made with agent-based models to study cell movement and cellular organization of the intestinal crypt. For this, Meineke et al used a lattice-free cylindrical surface to model experimental data showing that cell movement is a consequence of mitotic activity (Meineke, 2001). Taking cue of this, further research was done with spatial models of a colonic crypt, but now coupling the cell cycle with the mechanics of cell movement. The researchers used the model two hypotheses concerning stem cell behaviour: stem cells as immortal cells, and a stem cell governed through contact with Wingless-related integration site (WNT) molecules; providing evidence in support for the latter as more realistic scenario (Fletcher, 2012).

More recently, an agent-based model of cell death in the colon crypt showed novel emergent behaviour consistent with biological observations. The researchers reported on the localization of cell death to a small region of the top of the crypt (the mouth) as an emergent property in response to changes in either cell proliferation rates, corresponding with that observed in vivo. Further, they provide strong evidence that a cylindrical model can faithfully recapitulate biology better than a spherical one (Imgham-Dempster, 2017).

Colon crypts are of special interest because, although key cell biology has been recently discovered thanks to advances in cell detection technology, unfortunately, available and published data on crypt geometry and dynamics are very heterogeneous depending on the used methods. It is the consensus that the cell replacement comes from stem cells dividing at the bottom of the crypt, but the reported literature differs on the importance of the different metrics. Which is the biggest contributor to the time for monoclonality of a crypt: the geometry or the dynamics? The different theories about the culprit for fixation are based on data from static points in time during the crypt's evolution and they may seem incompatible at a first glance. Most data are available on the mouse small intestine; for which the number of stem cells has been estimated to range from four to sixteen (Kozar, 2013, Ritsma, 2014, Snippert, 2010). By the same token, the cell cycle time of stem cells is reported to range from $12 \mathrm{~h}$ to 28.5 hours (Gerike, 1998, Meineke, 2001, Ritsma, 2014, Bach, 2000, Escobar, 2010). Further, published values of cell cycle time of transiently amplifying cells range from 12 hours to 18 hours (Bach, 2000, Kozar, 2013, Li, 1994). These values affect the key hypothesis regarding the time it takes for a crypt to become monoclonal, and thus informing the onset of oncogenic mutations.

The main problem is that it is very difficult to track of all progeny of each cell, that can test these different hypotheses. For this we have built an agent-based model that is able to use the diverse parameters reported to reproduce the biological results and test the posited hypotheses to uncover the identity of the key contributor to the time for crypt fixation.

\section{Hypotheses on Intestine Crypt Evolution}

The human intestine can be divided into small and large intestine, the former forming the duodenum, the jejunum and the ileum whereas the latter is constituted by the caecum, the colon and the rectum. The inner lining of the intestine is composed by an epithelial monolayer with a high cell turnover. In all sections of the intestine, cell renewal starts at the bottom of invaginations, the intestinal crypts (Figure 1). The geometry and the cell composition of intestinal crypts vary in the different sections of the intestine (Buske, 2011), but a common feature is that epithelial cells are replenished by stem cells (SC) at the bottom which give rise to transit-amplifying cells (TAC) in the middle that divide for a certain time while they travel upwards trough the crypt. Once at the top, they terminally differentiate into different types of epithelial cells (EC) and either undergo apoptosis or are shed into the lumen of the intestine (Barker 2008).

The number and location of stem cells, the geometry of the crypt and the balance between SCs and TACs are tightly regulated in order to ensure homeostasis of the epithelial cells and the function of the intestine (Ritsma, 2014). This is mediated in part by two basal signalling molecules that extend from the bottom up: WNT molecules maintain a population of 
cells in a stem cell phenotype; while epidermal growth factor (EGF) molecules regulate cell proliferation. Both are maximum at the bottom and decrease in intensity as they go upwards along the crypt. Aberrations within these key molecules and their intracellular pathways have been implicated in colon carcinogenesis (Fletcher 2012).

It is believed that CRC starts with a mutation of one SC of one intestinal crypt and that the mutated cells expand within the crypt until it becomes composed of monoclonal cells. Monoclonality in intestinal crypts is then theorized to occur without the need of a proliferative advantage (Ritsma, 2014). However, the time to monoclonality offers a key metric for the successful establishment of mutations (Jemal, 2013). If a carcinogenic mutation occurs in a TA cell, the chances that this cell will be discarded before any more mutations are acquired is high, thus lowering the risk of carcinogenesis. However, if such a mutation occurs in a relatively stationary SC, then the likelihood that the mutation spreads to the whole crypt, i.e. fixated, is then high. To compound on the uncertainty, the role of heterogeneity and spatial structure in the evolution of the colon crypt is still a subject of intense debate (Stine, 2013). No clear pathway has been established in the development of colorectal cancer, and thus the contribution of the geometry of the colon crypt, the rate cell displacement and variation in the morphogens have to the time to monoclonality remains unknown (Simons, 2011). Once the mutation has become fixated however, new mutations can contribute to the oncogenic transformation with a similar fixation time as the one to the original monoclonality. The constraints that have been proposed as the affecting the fixation of mutations in the crypt hypothesised to be:

H1. The number of basal stem cell number. By determining the rate of mutation in vivo and combining this data with neutral-drift dynamics that describe intestinal stem cell replacement, the researchers found significantly lower numbers of SCs in the intestinal epithelium $(\sim 8$ in normal crypt, $\sim 12$ in adenoma) than those published in previous reports (Kozar, 2013); theorising a high impact on the time to monoclonality.

H2. The total number of stem cells regulated by extracellular influences. Evidence was put forward supporting the hypothesis that SCs are regulated by contact with WNT (Fletcher, 2012). It was then theorized that changes in the intensity of WNT would impact the fixation of mutations in the crypt.

H3. The proportion of side cell displacement. Experimental tracking of cell movements suggest that lateral division may displace basal SCs, thus becoming a key variable in the time to monoclonality (Snippert, 2010, Ritsma, 2014).

It is here where our a white-box cellular model can help by unifying the data and test the different theories to assess which of these hypotheses is valid and, if so, its range of validity.

\section{The System}

In order to investigate which of the hypotheses are valid, we created an agent-based model of the colon crypt. As described

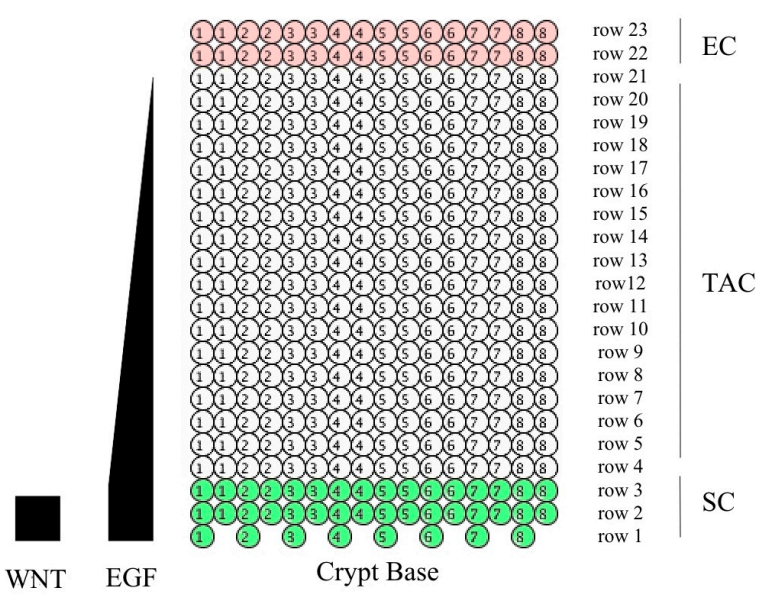

Figure 2: The compartments that make up the colon crypt model. The WNT gradient (right) maintains cells in SC state, while EGF regulates cell division. Cells divide from the bottom up, pushing or displacing other cells. Lineage is traced by numerically tagging each cell and their progeny.

in Figure 1, the colon crypt is an invagination that is in constant renewal. To simulate this tissue, we have designed a computational model that exhibits the same homeostatic behaviour of a healthy crypt. We have abstracted the behaviour at a cellular level and modelled each cell as a circular agent. For visual representation, we have adapted the invagination into a two-dimensional plane made up of cells with a continuous boundary to the left and right of the cells, thus preserving the original three-dimensional features (Figure 2). Cell differentiation, as in the biological system, is locally restricted by molecular signals that define the stem cell niche and the transit amplifying compartment: a WNT and a EGF gradient respectively (Smallbone, 2014). Regulated by these two gradients, EGF promotes proliferation while WNT keeps the cells in a stem state (Fletcher, 2012).

In our computational model, cells are represented by agents that react to the morphogens in the microenvironment, enabling cells to divide, quiesce or die (Figure 2). During normal homeostasis, three populations of morphogen-regulated cells coexist: Stem Cells (SCs) at the bottom, Transit Amplifying Cells (TACs) in the middle and fully differentiated Epithelial Cells (ECs) at the top (Figure 2). Cells are physically able to push other cells in all directions, with higher probability of pushing cells up or sideways up, a low probability of dividing sideways and an even lower probability of going downwards to the sides or downwards (Table 1). Stem cells proliferate at the bottom compartment, pushing cells up and supplying a fresh batch of TACs that eventually differentiate at the top of the crypt and are shed away. In this work we will focus on two key morphogens the process of cell renewal in the crypt: WNT (promoting the stem-cell phenotype) and EGF (promoting cell division and regulating cell differentiation). Both morphogens are maximum at the base of the mouse small intestine crypt and are thought to be provided by Paneth cells which reside at the bottom of the crypt (Sato, 2011). It is assumed that the morphogens concentrations decrease in a gradient throughout the length of the crypt (Bach, 2000). In the model, the WNT 
gradient, keeping all the cells in contact with it in a stem cell phenotype, is completely depleted 10 to $30 \mu \mathrm{m}$ (approximately one to three cell diameters) above the base. When cells are out of the WNT gradient, but still within the EGF gradient, they lose stem cell properties, start aging, and are able to divide proportionally to the bio-availability of EGF. Once these transit-amplifying cells are pushed outside of the EGF gradient (approximately $31 \mu \mathrm{m}$ above the base of the crypt) they become fully differentiated epithelial cells, stop dividing and their likelihood of being shed away or dying is $100 \%$. Besides EGF, other morphogens have been implicated in the regulation of TAC cells (Carulli, 2014) and EGF in the model is only representative of a putative morphogen acting by a gradient.

An additional component of the model is a restricted number of cell divisions within the TAC-compartment while the stem cells can divide indefinitely. It currently thought that TACs double only three to six times during crypt homoeostasis (Barker, 2008). This behaviour is simulated by a finite telomere length number which decreases by one with every cell division of a TAC cell and, when reaching zero, the cell loses its proliferative capacity. This relies on the biologic finding that only cells at the bottom of the intestine crypt express the telomerase protein (Hiyama, 2001). For long term maintenance of a crypt, the TAC cells of the first row above the stem cells have to harbour a minimal telomere length which allows enough cell divisions of TAC cells to replace the constant cell loss within a colon crypt (Montgomery, 2010).

\section{The Algorithm}

Every cell is queried at every time step in an asynchronous update. A random cell that has not been previously updated during the time step is picked and follows the update algorithm by which it is given the chance to decide whether to die, divide or do nothing per cell cycle (Figure 3 ). The cell identity is decided by its position with respect to the WNT gradient (SC) and the EGF gradient (TAC) though a probabilistic calculation. $\mathrm{SC}$ cells have a fixed rate of division, division $S C$, while under the influence of WNT. Their probability of division $100 \%$ throughout the SC compartment:

$$
P(\text { SCdiv })=100 *(\text { division } S C)
$$

TACs have $100 \%$ chance of dividing as they leave the WNT gradient but are still in contact with the EGF gradient. The TACs chance of division, TACdiv, decreases linearly to $0 \%$ as it travels to the top of the EGF gradient. To achieve this, we normalize the difference between the EGF and WNT, Agrad, and calculate the vertical distance, yPos, between the TAC cell and the end of the WNT gradient, which modulates division TAC:

\section{$P($ TACdiv $)=100 *($ divisionTAC $) *(y P o s-\Delta g r a d) / \Delta g r a d$}

Where divisionSC and divisionTAC are biological parameters shown in (Table 1). When a cell divides it pushes one of its neighbours, selected with the baseline probabilities shown in Table 1 . The probability of death is $100 \%$ when leaving the EGF gradient. We model aging as a decrease in telomere length, therefore reducing the number of times it can divide (initially 5 divisions) and persist within the TAC-compartment. Each round, the algorithm queries the system for a user defined condition. For this work we will use one of two user-specific

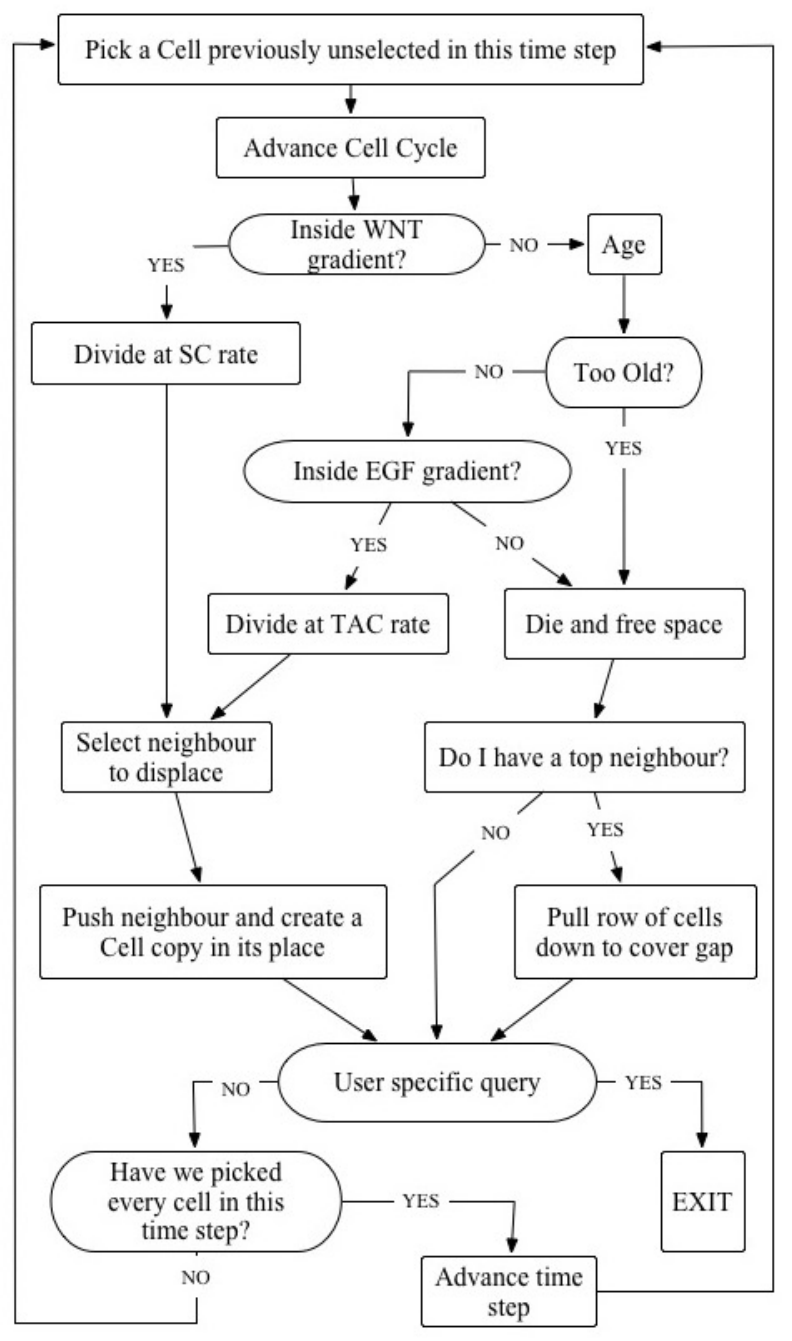

Figure 3: Algorithm for cell dynamics. The program ends when the user specific query (E.g. reaching monoclonality) is met.

queries: 1. a set time (in days), and 2. if the basal SCs have become monoclonal. If the user specific query is not met, it continues updating cells, and advancing to a new time step when every cell has been updated or stopping when the query has been met.

\section{Agent design}

We designed the agents to offer different information that could be displayed visually with regards to key metrics of interest. In the base visualization, SCs (cells inside the WNT Gradient) are tagged green, TACs (cells outside the WNT and in the EGF gradient) are marked white, and ECs (cells outside of both gradients) are coloured peach (Figure 2). To track the lineage of cells, we give a tag number to each of the initial basal SC which is inherited by their progeny throughout the simulation. In the initial baseline condition, as shown in Figure 2 we assume that cells that are in the column immediately on top of the SC are progeny of it and therefore inherit this number, which is displayed at the centre of each cell in the visualization. 


\begin{tabular}{|l|l|l|}
\hline Parameters & Value & Reference \\
\hline Cell Diameter & $10 \mu \mathrm{m}$ & Bach, 2000 \\
\hline Number of basal SC & 8 cells & $\begin{array}{l}\text { Kozar, 2013, } \\
\text { Ritsma, 2014 }\end{array}$ \\
\hline Total number of SC & $\begin{array}{l}12-16 \text { cells } \\
\text { (duodenum) }\end{array}$ & Snippert, 2010 \\
\hline $\begin{array}{l}\text { Width and length of } \\
\text { crypt }\end{array}$ & $\begin{array}{l}16 \mathrm{x} \\
25 \text { cells }\end{array}$ & $\begin{array}{l}\text { Bach, 2000, } \\
\text { Totafurno, 1988 }\end{array}$ \\
\hline $\begin{array}{l}\text { SC division rate } \\
\text { (divisionSC) }\end{array}$ & $\begin{array}{l}\text { Once every } \\
24 \text { hours max }\end{array}$ & Fischer, 2016 \\
\hline $\begin{array}{l}\text { TAC division rate } \\
\text { (divisionTAC) }\end{array}$ & $\begin{array}{l}\text { Once every } \\
12-24 \text { hours } \\
\text { max }\end{array}$ & Kozar, 2013 \\
\hline $\begin{array}{l}\text { Probability of Side } \\
\text { Displacement per } \\
\text { cell division (SDisp) }\end{array}$ & $\begin{array}{l}0.24 \text { (0.12 } \\
\text { each side) } \\
\text { to } \\
0.74\end{array}$ & Ritsma, 2014 \\
\hline $\begin{array}{l}\text { Probability of } \\
\begin{array}{l}\text { Downwards } \\
\text { Displacement } \\
\text { per cell division }\end{array}\end{array}$ & $\begin{array}{l}\text { TACs, } \\
0.00 \text { for SCs }\end{array}$ & Snippert, 2010 \\
\hline
\end{tabular}

Table 1: Baseline parameters used to simulate a murine small intestine crypt.

This gives a representation and a clear pathway of how cell mixing and eventually monoclonality occur. Other metrics such as the number of divisions and age are also stored in each agent and can be shown as the number displayed on each cell.

\section{Biological Parameters}

We have extracted key biological parameters from the literature and integrated them in the agent-based model, capturing the known effects of morphogens in the intestine crypt, while incorporating new ideas derived from (Fisher, 2016, Kozar, 2013, Ritsma, 2014). We ensure cells migrate from the bottom up with biologically-derived parameters shown in Table 1. To investigate the different hypotheses about the identity of the dynamical constraints that has the largest effect the fixation of mutations in the crypt, namely the number of basal stem cell number (Kozar, 2013), the total number of stem cells regulated by extracellular influences (Fletcher, 2012) or the proportion of side cell displacement (Ritsma, 2014), we have parametrized the model with baseline rates shown in Table 1 . These values have been published for the murine small intestine crypt, for which more reliable data are available compared to the murine colon and to the human intestine. One of the benchmarks and validation of our model is that homeostasis emerges from the interactions of the intestine crypt cells to the morphogens WNT and EGF (Figure 2).

\section{Model Validation 1: Positional Mitotic Index}

The essential mechanism responsible for the cell flux in the crypt is mitotic activity, which causes a pressure-driven passive movement from the bottom to the top of the crypt. Therefore, the ratio between the number of cells in a population undergoing mitosis to the total number of cells in a population can be used as a metric to assess the validity of the model to recapitulate known biology. For this, we will use positional mitotic index as our metric, as described in (Meineke, 2001).

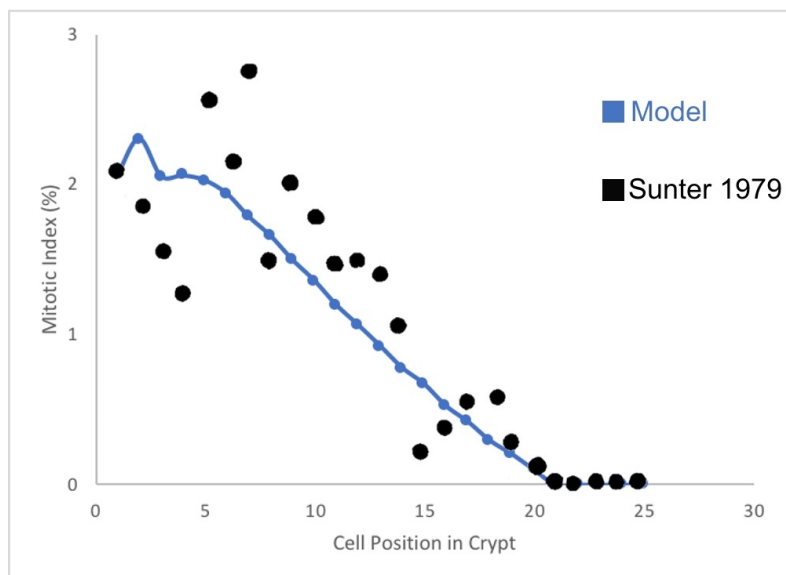

Figure 4. Mitotic index distribution in the crypt (upwards form the crypt as described in in Figure 2) of 100 simulated crypts with 8 basal and 16 suprabasal stem cells over a 30-day period. We compare it to data presented in Fig 4.iv of (Sunter, 1979).

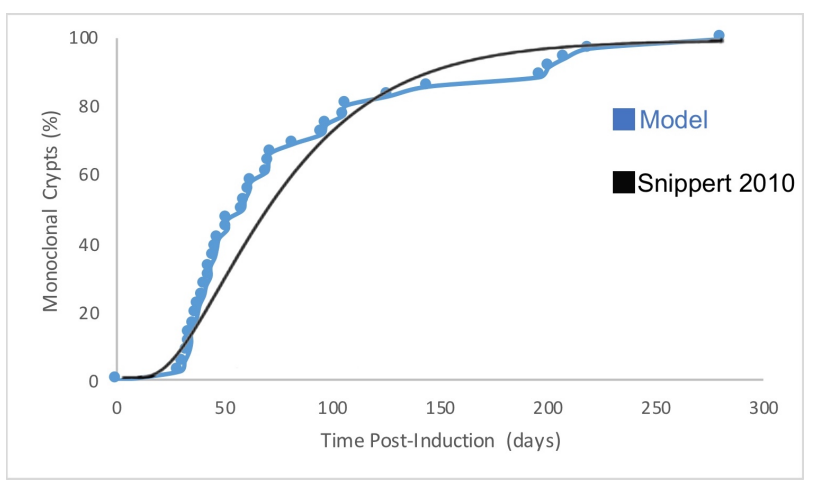

Figure 5. Frequency of monoclonal crypts over time as a percentage of surviving SC clones (out of initial 14) as predicted by neutral drift dynamics (Snippert, 2010).

In their work Sunter et al. show plots for the labelling index of cell proliferation within the large bowel of the mouse (Sunter, 1979). To validate our model, we ran 100 simulations and defined our end user-query as a 30-day period. When tested, the average division per row (Figure 4) recapitulates the data for mitotic index distribution in the crypt presented in Fig 4.iv of (Sunter, 1979), where there is a maximum of mitotic activity a few rows after leaving the base of the crypt and decreases throughout the rest of the rows.

\section{Model Validation 2: Neutral Drift Dynamics}

To provide evidence of a neutral drift in the cellular dynamics of intestinal stem cells, Snippert et al. collected clonal tracing data of stem cell loss. In their experimental system, a 14 basal stem cell population was calculated to have a probability of side displacement of 0.74 , demonstrating neutral competition amongst SCs. We have simulated 100 of such crypts (Figure 5) and found that our dynamics are in agreement with that reported in Figure 7D of (Snippert, 2010). This shows that the crypt dynamics are behaving as expected and that stem cell turnover follows a pattern of neutral drift dynamics of shortterm clonal analysis, where the basal stem cells double their numbers each day. 


\begin{tabular}{|l|l|}
\hline Number of basal SC 1 & 4 cells \\
\hline Number of basal SC 2 & 8 cells \\
\hline Number of basal SC 3 & 12 cells \\
\hline
\end{tabular}

Table 2: parameters used to investigate the role of basal SCs number to the time to monoclonality.

\begin{tabular}{|l|l|}
\hline WNT Gradient 1 & $30 \mu \mathrm{m}(100 \%)=20$ and 24 cells \\
\hline WNT Gradient 2 & $10 \mu \mathrm{m}(30 \%)=4$ and 8 cells \\
\hline
\end{tabular}

Table 3: parameters used to investigate the role of WNTregulated total SCC number to the time to monoclonality

\begin{tabular}{|l|l|}
\hline SDisp1 & $10 \%(5 \%$ each side $)$ \\
\hline SDisp2 & $20 \%(10 \%$ each side $)$ \\
\hline SDisp3 & $30 \%(15 \%$ each side $)$ \\
\hline SDisp4 & $40 \%(20 \%$ each side $)$ \\
\hline SDisp5 & $50 \%(25 \%$ each side $)$ \\
\hline
\end{tabular}

Table 4: parameters used to investigate the role of side displacement to the time to monoclonality.

\section{Experiments}

Our experiments test the constraints that are hypothesised to have the largest effect the fixation of mutations in the crypt:

H1. The number of basal stem cells (Kozar, 2013)

H2. The total number of stem cells regulated by extracellular influences (Fletcher, 2012)

H3. The proportion of side cell displacement (Ritsma, 2014)

The metric that we use to compare these hypotheses is the time it takes for 100 crypt simulations to reach monoclonality.

To investigate the different hypotheses, we first establish a baseline time to monoclonality, then create three experiments that simulate the parameters of each of the three proposed scenarios. We compare these results to the baseline time to identify the biggest contributor to the fixation of mutations in the crypt.

Experiment 1- Baseline time to monoclonality. In their celltracking experiments Ritsma et al have measured the time it takes for one basal SC sub-clone to divide sideways enough to make the entirety of the crypt a descendant of this cell, or monoclonal (Ritsma 2014). The researchers performed this experiment with a number of crypts and describe in their results the percentage of crypts that have become monoclonal in a 140day period. We perform 100 simulations with the baseline model based on the accepted parameters shown in Table 1, and measure our results using this same methodology.

Experiment 2- Role of basal SC number. In Experiment 2 we vary the total number of basal stem cells as hypothesised in (Kozar, 2013) as shown in Table 2.

Experiment 3- Role of the total number of stem cells regulated by extracellular influences. Here we test the extremes reported of the total number of stem cells regulated by extracellular influences as suggested by (Fletcher, 2012). We simulate the following parameters, see Table 3.

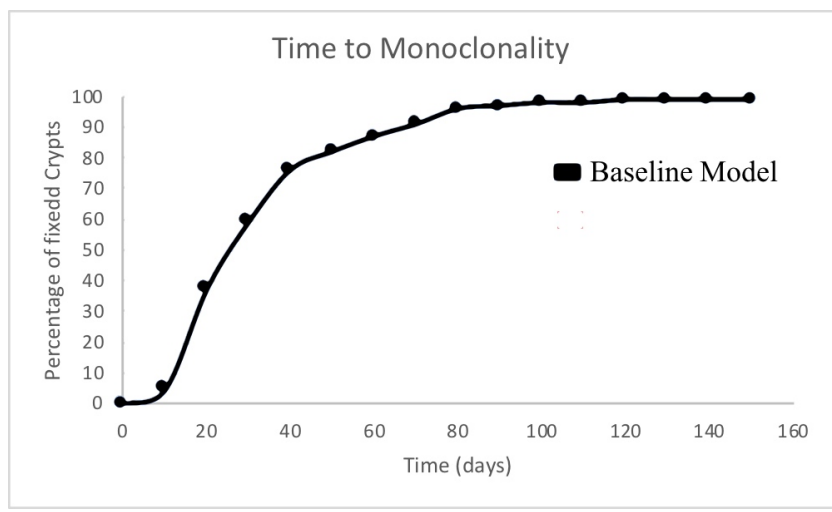

Figure 6: Simulation of biological data for monoclonality. From 100 simulations we measure every 10 days the percentage of simulations that have become monoclonal. By day 140, $100 \%$ have reached this state.

Experiment 4- Role of Side Displacement. Finally, we vary the probability of side cell displacement and quantify its contribution to the time to monoclonality, as suggested by (Ritsma, 2014) using the parameters shown in Table 4.

\section{Results}

\section{Results 1: Establishing a baseline time to monoclonality}

In our baseline simulations for monoclonality (Figure 6), 100\% of the crypts become monoclonal, or fixed, by day 140 following the same trend as that reported by Ritsma et al (Ritsma 2014). These dynamics are important to our understanding of the genetic evolution of the crypt, since they would give us an estimate of how fast we can expect a cancerous mutation to spread through a healthy crypt. Using this as our baseline, we will proceed to analyse the results of Experiments 2, 3 and 4.

\section{Results 2: Role of basal SC number}

Results from Experiment 2 shown in Figure 7 suggest that the number of basal SCs have a significant impact on the fixation of basal SCs on the crypt. Intuitively, having 4 SCs made the crypt converge quicker to monoclonality than 8SCs. Having $12 \mathrm{SCs}$, as you would find in an adenoma made the time to monoclonality much longer. The computational model predicts that the smaller the number of basal SCs the fastest it will become monoclonal.

\section{Results 3: Role of the total number of stem cells regulated by extracellular influences}

Not so intuitively, the total number of SCs did not seem to affect the time to monoclonality as much, as can be seen in Figure 8, where the time to monoclonality persists even if the WNT is lowered to $30 \%$. These simulations suggest that the total number of SCs, mediated by WNT intensity, plays a lesser role in the time to monoclonality than the total number of basal SCs (Figure 8). 


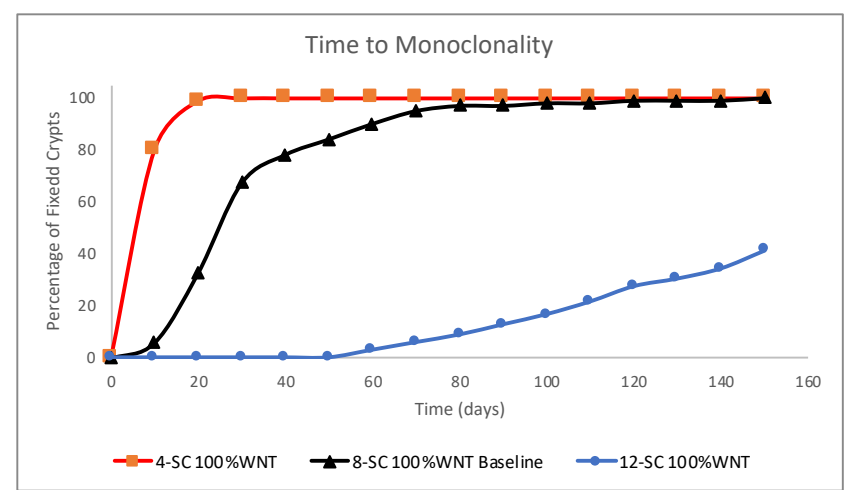

Figure 7: Results from Experiment 2 show that the variation in basal SC number has a strong impact on monoclonality.

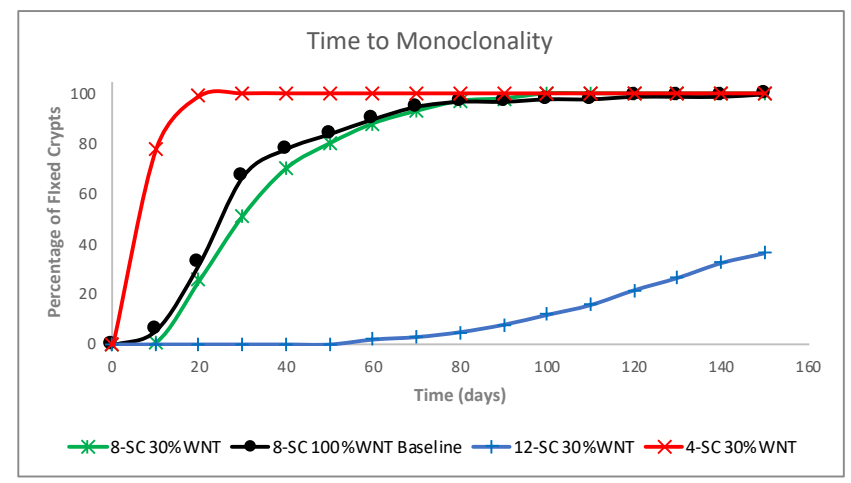

Figure 8: Results from Experiment 3; the variation in total SC number mediated by WNT intensity.

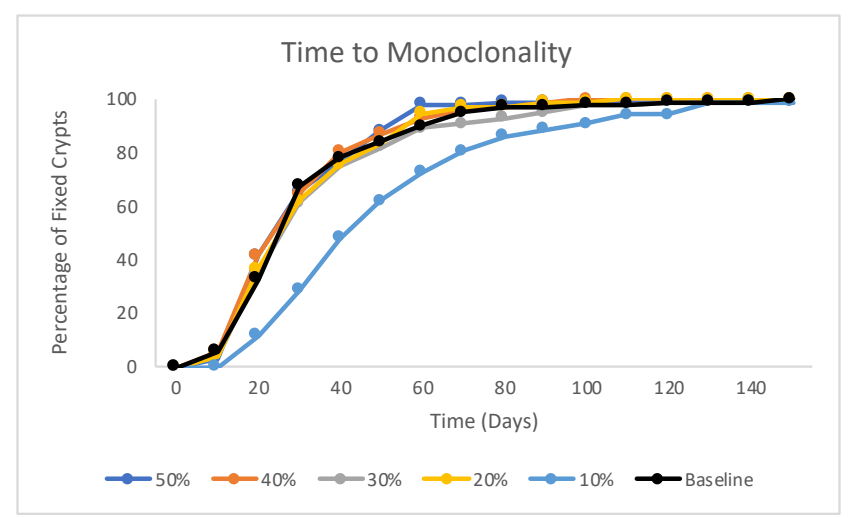

Figure 9. Results from Experiment 4 (variation in sideways displacement). We varied the probability of effective sideways division, with equal probability of displacing neighbours on each side. Results suggest that there is a saturation point that maximises time to monoclonality.

\section{Results 4: Role of Side Displacement}

The probability that a cell will divide sideways, displacing the neighbours that compete with them for genetic dominancy in the crypt has an intuitively profound impact on the time to monoclonality. Results from Experiment 4, as shown on Figure 9 , suggest that there is a saturation point in which increasing the probability of side displacement aids in the fixation of a given genotype in the crypt. After reaching $20 \%$ probability of sideways displacement, the basal SCs fiercely compete with each other for genetic dominance. Side displacement exceeding $20 \%$ seems to hit a ceiling when it comes to genetic dominance, so further increase in side displacement makes little difference on the time to fixation. This evidence points to intricate, nonlinear dynamics of competition due to side displacement that play a role on most cases for the fixation of a $\mathrm{SC}$, larger than the effect of WNT-mediated SC total; but not as large as the number of basal SC.

In summary, the evidence provided by the model indicates that all three hypotheses are supported by evidence, but the contribution to the proposed hypotheses rank in terms of the highest to lowest magnitude as follow:

1: The number of basal stem cell number (Kozar, 2013)

2: The proportion of side cell displacement (Ritsma, 2014)

3: The total number of stem cells regulated by extracellular influences (Fletcher, 2012)

\section{Conclusions}

We have created a model that faithfully models cell-cell and cell-morphogen interaction in colonic crypts. Our model recapitulates reported data from murine experiments, exhibits the known crypt biological behaviour and can be altered to simulate diverse realistic scenarios to answer key questions regarding crypt dynamics. We have investigated hypotheses that show significant variation in the reported literature, but that can be unified under an ALife model. To identify the source of the highest impact to the fixation of mutations in the colon crypt (the time to monoclonality) we have systematically varied the number of basal and total SCs, as well as the probability of their sideways physical division. The computational model shows that the smaller the basal number of stem cells the fastest it will become monoclonal, but the overall number of SCs has little impact on this. Further, the model suggests that there is a saturation point of side-displacement, past which intense competition between the dividing cell for genetic dominance yields very similar outcomes.

In our simulations, the change in total number of stem cells regulated by extracellular influences as suggested by (Fletcher, 2012) had the smallest impact in the time to monoclonality. Our results suggest that the proportion of side cell displacement plays a more important role, as proposed by (Ritsma, 2014), but in this research we showed that there is a regime of validity for this impact. Finally, our results show the number of basal stem cell number had the highest impact to reaching monoclonality faster, as proposed by (Kozar, 2013). This has important repercussions for the spread and fixation of mutations in crypts and sheds some light on a realistic quantification on the rate of oncogenic transformation.

\section{Future Work}

We will focus on in silico experiments that cannot be done in vitro or in vivo, but which have a significant impact on the time to monoclonality such as the rate of downward cell movement; changes in the intensity of the gradients EGF and WNT and, importantly, the crypt survival dependence on the stem cells. Furthermore, we anticipate that refinement on the internal genome with realistic signalling pathways and opportunities for 
their disruption will shed light on current research on aging and cancer initiation.

We have presented a model that helps bridge some of the diverse in vitro and in vivo murine experiments reported in the literature, and we believe that the next step is to further extend this bridge to clinically-relevant human data for therapeutic discoveries.

\section{Acknowledgments}

We would like to thank the Integrated Mathematical Oncology group at Moffitt Cancer Center, Braintree Ltd and UCL for fruitful discussions, and facilitating this collaborative research.

\section{References}

Andrews, P. S., Polack, F. A., Sampson, A. T., Stepney, S., \& Timmis, J. (2010). The CoSMoS process, version 0.1: A process for the modelling and simulation of complex systems. University of York Technical Report YCS-2010, 453.

Araujo, A., Bentley, P. J., \& Baum, B. (2010). Modelling the Role of Aneuploidy in Tumour Evolution. In ALIFE (pp. 488-495).

Bach, S. P., Renehan, A. G., \& Potten, C. S. (2000). Stem cells: the intestinal stem cell as a paradigm. Carcinogenesis, 21(3) 469-476.

Barker N, van de Wetering M, Clevers H. The intestinal stem cell. Genes Dev. 2008 Jul 15;22(14):1856-64.

Bentley, K. (2013) "Artificial life in the fight against cancer," IEEE Symposium on Artificial Life (ALife), Singapore, pp. 145-147.

Bravo, R., \& Axelrod, D. E. (2013). A calibrated agent-based computer model of stochastic cell dynamics in normal human colon crypts useful for in silico experiments. Theoretical Biology and Medical Modelling, 10(1), 66.

Buske, P., Galle, J., Barker, N., Aust, G., Clevers, H., \& Loeffler, M. (2011). A comprehensive model of the spatio-temporal stem cell and tissue organisation in the intestinal crypt. PLoS computational biology, 7(1), e1001045.

Carulli AJ, Samuelson LC, Schnell S. (2014) Unraveling intestinal stem cell behavior with models of crypt dynamics. Integr Biol (Camb). 6(3):243-57.

Escobar M, Nicolas P, Sangar F, Laurent-Chabalier S, Clair P, Joubert D, Jay P, Legraverend C. (2011) Intestinal epithelial stem cells do not protect their genome by asymmetric chromosome segregation. Nat Commun. 2:258.

Fischer, J. M., Calabrese, P. P., Miller, A. J., Muñoz, N. M., Grady, W. M., Shibata, D., \& Liskay, R. M. (2016). Single cell lineage tracing reveals a role for TgfßR2 in intestinal stem cell dynamics and differentiation. Proceedings of the National Academy of Sciences, 113(43), 12192-12197.

Fletcher, Alexander G., Christopher JW Breward, and S. Jonathan Chapman. (2012) Mathematical modeling of monoclonal conversion in the colonic crypt. Journal of theoretical biology $300: 118-133$.

Fontana, A. (2010). An Artificial Life Model for Carcinogenesis. ALIFE (pp. 101-108).

Froese, T., Virgo, N., \& Ikegami, T. (2014). Motility at the origin of life: Its characterization and a model. Artificial life, 20(1), 55-76.

Galle, J., Hoffmann, M., \& Aust, G. (2009). From single cells to tissue architecture-a bottom-up approach to modelling the spatio-temporal organisation of complex multi-cellular systems. Journal of mathematical biology, 58(1-2), 261.

Gerike TG, Paulus U, Potten CS, Loeffler M. (1998) A dynamic model of proliferation and differentiation in the intestinal crypt based on a hypothetical intraepithelial growth factor. Cell Prolif. 31(2):93-110.

Hiyama E, Hiyama K, Yokoyama T, Shay JW. (2001) Immunohistochemical detection of telomerase (hTERT) protein in human cancer tissues and a subset of cells in normal tissues. Neoplasia. 3(1):17-26.
Ingham-Dempster, T., Walker, D. C., \& Corfe, B. M. (2017). An agentbased model of anoikis in the colon crypt displays novel emergent behaviour consistent with biological observations. Royal Society open science, 4(4), 160858.

Jemal, A., Center, M. M., DeSantis, C. \& Ward, E. M. (2010) Global patterns of cancer incidence and mortality rates and trends. Cancer Epidemiol. Biomarkers Prev. 19, 1893-1907.

Kalmykov, L. V., \& Kalmykov, V. L. (2015). A solution to the biodiversity paradox by logical deterministic cellular automata. Acta biotheoretica, 63(2), 203-221.

Kauffman, S. A. (2011). Approaches to the Origin of Life on Earth. Life, $1(1), 34-48$.

Kozar, S., Morrissey, E., Nicholson, A. M., van der Heijden, M., Zecchini, H. I., Kemp, R., ... \& Winton, D. J. (2013). Continuous clonal labeling reveals small numbers of functional stem cells in intestinal crypts and adenomas. Cell stem cell, 13(5), 626-633.

Lenaerts, T., Giacobini, M., Bersini, H., Bourgine, P., Dorigo, M., \& Doursat, R. (2011). Advances in Artificial Life, ECAL 2011.

Li YQ, Roberts SA, Paulus U, Loeffler M, Potten CS. (1994) The crypt cycle in mouse small intestinal epithelium. J Cell Sci. 107( Pt 12):3271-9.

Meineke, F. A., Potten, C. S., \& Loeffler, M. (2001). Cell migration and organization in the intestinal crypt using a lattice-free model. Cell proliferation, 34(4), 253-266.

Montgomery RK, Carlone DL, Richmond CA, Farilla L, Kranendonk ME, Henderson DE, Baffour-Awuah NY, Ambruzs DM, Fogli LK, Algra S, Breault DT. (2011) Mouse telomerase reverse transcriptase (mTert) expression marks slowly cycling intestinal stem cells. Proc Natl Acad Sci U S A. 108(1):179-84.

Rejniak, K. A., \& Anderson, A. R. (2011). Hybrid models of tumor growth. Wiley Interdisciplinary Reviews: Systems Biology and Medicine, 3(1), 115-125.

Ritsma, L, et al. (2014) Intestinal crypt homeostasis revealed at singlestem-cell level by in vivo live imaging. Nature 507 , no. $7492: 362-$ 365.

Rübben, A., \& Nordhoff, O. (2012). A systems approach defining constraints of the genome architecture on lineage selection and evolvability during somatic cancer evolution. Biology open, BIO20122543.

Sato T, van Es JH, Snippert HJ, Stange DE, Vries RG, van den Born M, Barker N, Shroyer NF, van de Wetering M, Clevers H. (2011) Paneth cells constitute the niche for Lgr5 stem cells in intestinal crypts. Nature. 469(7330):415-8

Simons, B. D. \& Clevers, H. (2011) Strategies for homeostatic stem cell self-renewal in adult tissues. Cell 145, 851-862.

Smallbone, K., \& Corfe, B. M. (2014). A mathematical model of the colon crypt capturing compositional dynamic interactions between cell types. International journal of experimental pathology, 95(1), 1-7.

Snippert HJ, van der Flier LG, Sato T, van Es JH, van den Born M, KroonVeenboer C, Barker N, Klein AM, van Rheenen J, Simons BD, Clevers H. (2010) Intestinal crypt homeostasis results from neutral competition between symmetrically dividing Lgr5 stem cells. Cell. 143(1):134-44.

Stine,R.R. \& Matunis,E.L . (2013) Stem cell competition: finding balance in the niche. Trends Cell Biol. 23, 357-364.

Sunter, J. P., Appleton, D. R., De Rodriguez, M. S., Wright, N. A., \& Watson, A. J. (1979). A comparison of cell proliferation at different sites within the large bowel of the mouse. Journal of anatomy, 129(Pt 4), 833 .

Sütterlin, T., Huber, S., Dickhaus, H., \& Grabe, N. (2009). Modeling multicellular behavior in epidermal tissue homeostasis via finite state machines in multi-agent systems. Bioinformatics, 25(16), 20572063.

Swanson, K. R., Bridge, C., Murray, J. D., \& Alvord, E. C. (2003). Virtual and real brain tumors: using mathematical modeling to quantify glioma growth and invasion. Journal of the neurological sciences, 216(1), 1-10.

Totafurno J, Bjerknes M, Cheng H. (1988) Variation in crypt size and its influence on the analysis of epithelial cell proliferation in the intestinal crypt. Biophys. 54(5):845-58. 